\title{
A comparative study of cord blood haemoglobin and ferritin in normotensive versus preeclamptic patients
}

\author{
Ratna Kanta Talukdar, Sasindra Kumar Das, Indira Dokania
}

\author{
Corresponding author: Dr. Sasindra Kumar Das, MD, Assistant Professor, Department of \\ Obstetrics and Gynaecology, Gauhati Medical College and Hospital, Guwahati, 781032, Assam,, \\ India; Email : sasindradas@gmail.com
}

Distributed under Attribution-Non Commercial - Share Alike 4.0 International (CC BY-NC-SA 4.0)

\begin{abstract}
Background: During pregnancy, newborn development is directly affected by mother's well being. In India pregnancy induced hypertension incidence corresponds to be around $15 \%$. The babies born to hypertensive mother have higher incidence of haematological changes. Due to uteroplacental vascular insufficiency or due to chronic foetal hypoxia there is increased iron utilization during enhanced erythropoiesis. Objectives: The aim is to compare the cord blood haemoglobin and ferritin level in newborns of normotensive versus preeclamptic patients. Materials and methods: It is a hospital based prospective case control study conducted from June 1st 2019 to 31 st May 2020 for a period of one year. Over the study period, total 200 patients are included in the study and analysis of cord blood haemoglobin and ferritin level of 100 preeclamptic (case) and 100 normotensive (control) mothers were done. All data were analysed using SPSS version 21. A p - value $<0.05$ is considered as statistically significant at $5 \%$ level of significance. Results: Out of 100 preeclamptic patients $96 \%$ were booked cases and their mean age was $24.34 \pm 3.77$ years and out of 100 normotensives patients $99 \%$ were booked and their mean age was $23.71 \pm 3.72$ years. Haemoglobin level were found to be significantly higher in the preeclamptic group (case) as compared to normal group (control) with $p$ value of $<0.004$ and mean value of 16.91 and 17.39 in control and cases respectively. In case of ferritin, the level were found to be significantly lower in the preeclamptic group as compared to normal group with $\mathrm{p}$ value $<0.0001$ and mean value of 158.94 and 76.98 in control and cases respectively. Conclusion: The chronic intrauterine hypoxia caused by preeclampsia causes significant changes in the haematological values in these babies.
\end{abstract}

Keywords: Preeclampsia, cord blood haemoglobin, cord blood ferritin, normotensive.

Hypertensive disorders are the most common cause of maternal and foetal mortality and morbidity ${ }^{1-3}$. In India pregnancy induced hypertension incidence corresponds is around $15 \%{ }^{1}$. Pre eclampsia is a multisystem disorder of the mother in which foetus is affected because of uteroplacental insufficiency ${ }^{4}$ as due to insufficient invasion and ingrowth of the trophoblasts that inhibit vessel dilation, thus reducing maternal blood supply to the intervillous space, and thus reducing perfusion and causing hypoxia, therefore releasing some toxin causing vascular endothelial dysfunction and damage leading to preeclampsia. The babies born to hypertensive mother have higher incidence of somatic growth retardation, low Apgar scores, and have a spectrum of haematological changes ${ }^{4,5}$ which include polycythemia, thrombocytopenia, leukopenia, neutropenia.

Iron is a essential micronutrient that is vital for early brain growth and function since it supports neuronal and glial metabolism, neurotransmitter synthesis and myelination ${ }^{6}$. Newborn infants with the lowest quartile of cord ferritin concentrations $(<76 \mathrm{ug} / \mathrm{l})$ have impaired mental and psychomotor function at school age ${ }^{7}$. The major form of iron storage is ferritin. Factors that influence neonatal ferritin concentration at birth include gestational age and conditions altering maternal-foetal iron exchange. Due to uteroplacental

Received: $20^{\text {th }}$ December 2020, Peer review completed: $28^{\text {th }}$ February 2021, Accepted: $7^{\text {th }}$ March 2021.

Talukdar RK, Das SK, Dokania I. A comparative study of cord blood haemoglobin and ferritin in normotensive versus preeclamptic patients. The New Indian Journal of OBGYN. 2021; 8(1): 23-6. 
vascular insufficiency or due to chronic foetal hypoxia there is increased iron utilization during enhanced erythropoiesis ${ }^{8}$. According to some studies the haemoglobin level was found to be higher in some cases and also lower in other studies ${ }^{4,5,9}$.

Keeping in mind the various changes that has been occurring in foetus born to preeclamptic mothers like haemoglobin and ferritin level, a comparative study has been proposed regarding the effect of pre eclampsia in the levels of haemoglobin and ferritin in neonates by taking samples from umbilical cord of foetus born to preeclamptic and normotensive pregnant women admitted in department of obstetrics and gynaecology, Gauhati Medical College and Hospital in a 1 year study period. The aim is to compare the cord blood haemoglobin and ferritin of babies born to normotensive patients versus preeclamptic patients.

\section{Methodology}

A hospital based prospective case-control study, conducted in department of obstetrics and gynaecology, Gauhati medical college and hospital, Guwahati, Assam from June 1st 2019 to 31st May 2020 for a period of one year. Permission for the study was obtained from the ethical committee. It was carried out on 200 patients meeting the inclusion and exclusion criteria, who were admitted to department of obstetrics and gynaecology. Out of these, 100 patients had preeclampsia (case) and 100 were normotensive (control) patients. Pregnant female with preeclampsia with other comorbid conditions like essential hypertension, diabetes, renal disease, liver disease, anemia and also pregnant females below 18years and more than 35years were excluded from the study.

Data of the patients were collected in a proforma from time to time. Results obtained from the study of cord blood were recorded. Detailed history was recorded including complete demographic details, dietary history, past medical

history, previous obstetric history, and antenatal Table 2: Mean distribution, standard deviation (SD), mean difference and p-value history including details of any antenatal of cord blood haemoglobin.

complications.

The case group included 100 pregnant women Cord blood $\mathrm{Hb}$

whose systolic blood pressure was $>140 \mathrm{mmHg}$ and diastolic pressure $>90 \mathrm{mmHg}$ or both in a woman known to be normotensive prior to pregnancy. The diagnosis was made if two such abnormal blood pressure measurements were recorded at least 6 hours apart. The first and fifth Korotkoff sounds were used to determine the systolic and diastolic components respectively. Preeclampsia was diagnosed when along with gestational hypertension there was presence of proteinuria and / or presence of features suggestive of end organ damage like thrombocytopenia, oliguria, epigastric or upper quadrant abdominal pain, elevated liver enzymes , headache and visual disturbances.

$2 \mathrm{ml}$ of cord blood anticoagulated with EDTA and in a clot vial were collected from these babies and haemoglobin and ferritin level were estimated. For haemoglobin estimation, cyanmeth method was used and for ferritin estimation, chemiluminescence immunoassay was used.

Chi square or Fischers exact test was used to evaluate difference between categorical variables. Data were checked for normality using Kolmogorov - Smirnova and Shapiro Wilk test. Independent t-test was used depending on fulfilment of normality assumption for mean difference or Man Whitney test was used for normal data. All data were analysed using SPSS version 21. A p - value $<0.05$ was considered as statistically significant at $5 \%$ level of significance.

\section{Results}

During the study period, 100 preeclamptic and 100 normotensive patients with maternal age between 18-35 years and gestational age between 36-42 weeks were included in the study group and control group respectively.

\begin{tabular}{lll}
\hline Parameters & Cases $(\mathbf{n}=\mathbf{1 0 0})$ & Control $(\mathbf{n}=\mathbf{1 0 0})$ \\
\hline Maternal age in years & $24.34 \pm 3.77$ & $23.71 \pm 3.72$ \\
Booked cases & $96 \%$ & $99 \%$ \\
Mean systolic BP in mmHg & $158 \pm 11.27$ & $113.98 \pm 8.52$ \\
Mean diastolic BP in mmHg & $102.3 \pm 9.2$ & $73.18 \pm 7.23$ \\
Gestational age in weeks & $38.94 \pm 1.46$ & $39.16 \pm 1.39$ \\
\hline
\end{tabular}

The important factors relating to pregnancy of hypertensive and normotensive mothers are given in table 1 .

A haemoglobin value of less than $13.5 \mathrm{~g} / \mathrm{dl}$ was considered anaemic and a level more than $22 \mathrm{~g} / \mathrm{dl}$ was considered polycythemic. Comparisons of cord blood haemoglobin among the newborns of normotensive versus

\begin{tabular}{lllll}
\hline Parameter & $\begin{array}{l}\text { Control }(\mathbf{n}=\mathbf{1 0 0}) \\
\text { Mean } \pm \text { SD }\end{array}$ & $\begin{array}{l}\text { Cases }(\mathbf{n}=\mathbf{1 0 0}) \\
\text { Mean } \pm \text { SD }\end{array}$ & $\begin{array}{l}\text { Mean } \\
\text { difference }\end{array}$ & P-value \\
\hline Cord blood $\mathrm{Hb} \%$ & $16.91 \pm 1.07$ & $17.39 \pm 1.25$ & -0.480 & 0.004 \\
\hline
\end{tabular}
preeclamptic patients are shown in table 2. Haemoglobin level were found to be significantly higher in the study group as compared to control group ( $\mathrm{p}<0.004)$.

In the present study, when comparing umbilical cord blood data from normal and pre-eclamptic pregnancies (table 3), we found that ferritin level were found to be significantly lower in the study group as compared to control group $(\mathrm{p}<0.0001)$. 
The New Indian Journal of OBGYN. 2021 (July-December);8(1)

Table 3: Mean distribution, standard deviation (SD), mean difference and $p$ - value of cord blood ferritin

\begin{tabular}{lllllll}
\hline Parameter & $\begin{array}{l}\text { Control }(\mathbf{n}=100) \\
\text { Mean } \pm \text { SD }\end{array}$ & $\begin{array}{l}\text { Cases }(\mathbf{n}=\mathbf{1 0 0}) \\
\text { Mean } \pm \text { SD }\end{array}$ & $\begin{array}{l}\text { Mean } \\
\text { difference }\end{array}$ & Lower & Upper & P-value \\
\hline Cord blood ferritin & $158.94 \pm 57.18$ & $76.98 \pm 64.48$ & 81.96 & 64.96 & 98.95 & $<0.0001$ \\
\hline
\end{tabular}

When preeclampsia was distributed on the basis of severity it was seen that around 17 preeclamptic mothers had mild preeclampsia and 83 had severe preeclampsia. It was seen that cord blood haemoglobin when compared with the severity of preeclampsia, there was no statistical significance seen with p-value being 0.579 . Similarly when cord blood ferritin level was compared with the severity of preeclampsia, there was no statistical significance seen with p-value being 0.559 (table 4).

Table 4: Comparison of cord blood haemoglobin and ferritin level with severity of preeclampsia

\begin{tabular}{llll}
\hline Parameters & & Mean distribution & P - value \\
\hline Cord blood & Mild & $17.24 \pm 1.147$ & 0.579 \\
haemoglobin & Severe & $17.42 \pm 1.279$ & \\
\hline Cord blood & Mild & $85.35 \pm 54.526$ & 0.559 \\
ferritin & Severe & $75.27 \pm 66.495$ & \\
\hline
\end{tabular}

\section{Discussion}

Hypertensive disorders, one of the most common complications in obstetrical field. Hypertensive disorders especially preeclampsia is a major cause of maternal and perinatal morbidity and mortality worldwide. In our study, the haemoglobin level in cord blood of the study group was found to be significantly higher than the control group with p-value being $<0.004$. Some studies showing the similar result are: Kurlat et al (1992) ${ }^{10}$ - The risk of polycythemia was shown to be 12.6 fold higher in babies of hypertensive mothers compared to the general population similar to Huang SC, Chang FM et al (1994) ${ }^{11}$ which also shows elevated haemoglobin $(\mathrm{p}<0.05)$ in preeclamptic pregnancies. Bolat A et al ${ }^{9}$ and Evalyn Singnarpi et al ${ }^{2}$ also shows that risk of polycythemia was higher in babies of hypertensive mothers compared to the general population with significance value $<0.05$. But according to Sivakumar et al ${ }^{4}$ and Prekshya et $\mathrm{al}^{5}$ there was no significant differences in haemoglobin values which was similar to Mosayebi $\mathrm{Z}$ et al ${ }^{12}$, Naim Eman et al ${ }^{1}$ and Catarino et al ${ }^{13}$ who found similar values for $\mathrm{Hb}$ values.

Our study demonstrates that babies born to pre-eclamptic patients who were at risk of for impaired uteroplacental blood flow had depressed ferritin levels. The AGA infants of mothers with preeclampsia also had increased serum transferrin levels with low serum ferritin values. According to Brunner et $\mathrm{al}^{14}$, Dewolf $\mathrm{F}$ et al ${ }^{15}$, in preeclampsia vasospasm, endothelial deposition and lipid deposition in the uteroplacental arterioles leads to compromised transport of

\section{References}

iron to the foetus. In the present study, when comparing umbilical cord blood data from normal and pre-eclamptic pregnancies, we found that ferritin level were found to be significantly lower in the study group as compared to control group $(\mathrm{p}<0.0001)$. Chockalingam et al ${ }^{16}$ and Siddappa et al ${ }^{17}$ shows the similar result that infants of pre-eclamptic mothers had significantly lower ferritin levels than did asymptomatic gestational age matched control infants without these conditions. Some studies shows the contradictory result like Bolat A et al ${ }^{9}$ who found Ferritin levels to be higher in the newborns of hypertensive mothers compared to control group. While Evalyn Singnarpi et al ${ }^{2}$ found no difference in ferritin levels between the two groups.

\section{Conclusion}

We conclude that the cord blood haemoglobin of newborn of preeclamptic are higher and ferritin levels are lower as compared to newborns of normotensive patients. This parameters can be used for the screening purpose to detect the haematological changes in newborns in order to attempt to provide these babies with decreased morbidity and improved growth, development and survival.

Acknowledgments: The authors express their sincere appreciation to the administration of Srimanta Sankaradeva University of health sciences for giving their approval and assistance in conducting the research. In addition, the authors are grateful to the mothers who participated willingly in this study.

\section{Conflict of interest: None. Disclaimer: Nil.}

1. Eman N, Manazir AS, Uzma F, Nasreen N. Comparative study of cord blood hematological profile of neonates born to mothers with and without pregnancy-induced hypertension: A prospective case control study. Indian Journal of Child Health. 2017 Nov 22: 554-60.

2. Singnarpi E, Bhattacharjee D, Baidya JL, Jamatia SNN. Study of changes in haemoglobin level and leukocyte counts in cord blood of newborns of hypertensive mothers in AGMC and GB pant hospital. J Evol Med Dent Sci. 2014: 3(63): 13905-12.

3. Catarino C, Rebelo I, Belo L, Quintanilha A, SantosSilva A. Umbilical cord blood changes in neonates from a preeclamptic pregnancy. From preconception to postpartum. 2012 Mar 23; 2012: 269-87. 
The New Indian Journal of OBGYN. 2021 (July-December);8(1)

4. Sivakumar S, Bhat BV, Badhe BA. Effect of pregnancy induced hypertension on mothers and their babies. The Indian Journal of Pediatrics. 2007 Jul 1; 74(7): 623-5.

5. Prakash PL, Kumar PS, Murthy MV, Haricharan KR. Assessment of hematological profile of newborn at birth, born to mothers with gestational hypertension, preeclampsia and eclampsia syndrome. Journal of Evolution of Medical and Dental Sciences. 2013 Aug 26; 2(34): 6360-70.

6. Dallman PR. Biochemical basis for the manifestations of iron deficiency. Ann Rev Nutr. 1986: 6 13-40.

7. Tamura T, Goldenberg R, Hou J, Johnston F, Cliver SP, Ramsey SL, et al. Cord serum ferritin concentrations and mental and psychomotor development of children at five years of age. J pediatr 2002; 140: 165-70.

8. Finne PH. Erythropoietin levels in the cord blood as an indicator of intrauterine hypoxia. Acta Paediatr Scand. 1966; 55: 478-88.

9. Bolat A, Gursel O, Kurekci E, Atay A, Ozcan O. Blood parameters changes in cord blood of newborns of hypertensive mothers. Eurj Pediatr. 2013; 172: 1501-9.

10. Kurlat I, Sola A. Neonatal polycythemia in appropriately grown infants of hypertensive mothers . Acta Paediatr. 1992; 81: 662-4.

11. Huang SC, Chang FM. The adverse effect on fetal hemogram by preeclampsia: marked anisocytosis with normocytic, normochromic erythrocythemia as well as thrombocytopenia. Early human development. 1994; 37(2): 91-8.

12. Mosayebi Z, Nariman S, Hosseini L, Moyahedin AH. Evaluation of laboratory disorders in admitted neonates in NICU who were born to pre-eclamptic mothers. J Comprehensive Pediatr. 2013; 3(5): 194-9.

13. Catarino C, Rebelo I, Belo L, Rocha-Pereira P, Rocha S, Castro EB, et al. Erythrocyte changes in preeclampsia: relationship between maternal and cord blood erythrocyte damage. Journal of perinatal medicine. 2009 Jan 1; 37(1): 19-27.

14. Brunner HR, Gavras H. Vascular damage in hypertension. Hopsp Pract. 1975; 10: 97-105.

15. DeWolf F, Robertson WB, Brosen I. The ultrastructure of acute atherosis in hypertensive pregnancy. Am J Obstet Gynecol. 1975;123: 164-74.

16. Chockalingam UM, Murphy E, Ophoven JC, Weisdorf $\mathrm{SA}$, Georgieff MK. Cord transferrin and ferritin values in newborn infants at risk for prenatal uteroplacental insufficiency and chronic hypoxia. J pediatr. 1987 Aug 1; 111(2): 283-6.

17. Siddappa AM, Rao R, Long JD, Widness JA, Georgieff MK. The assessment of newborn iron stores at birth: a review of the literature and standards for ferritin concentrations. Neonatology. 2007; 92(2): 73-82.

\footnotetext{
Ratna Kanta Talukdar ${ }^{1}$, Sasindra Kumar Das ${ }^{2}$, Indira Dokania $^{3}$

${ }^{1}$ Professor, Department of Obstetrics and Gynaecology, Gauhati Medical College and Hospital, Guwahati, Assam, India; ${ }^{2}$ Assistant Professor, Department of Obstetrics and Gynaecology, Gauhati Medical College and Hospital, Guwahati, Assam, India;

${ }^{3}$ Post Graduate Trainee, Department of Obstetrics and Gynaecology, Gauhati Medical College and Hospital, Guwahati, Assam, India.
} 